\title{
Spatial and temporal variability of the concentration field from localized releases in a regular building array
}

Article

Accepted Version

Goulart, E. V., Coceal, O., Branford, S., Thomas, T. G. and Belcher, S. E. (2016) Spatial and temporal variability of the concentration field from localized releases in a regular building array. Boundary-Layer Meteorology, 159 (2). pp. 241-257. ISSN 1573-1472 doi: https://doi.org/10.1007/s10546-0160126-0 Available at https://centaur.reading.ac.uk/60487/

It is advisable to refer to the publisher's version if you intend to cite from the work. See Guidance on citing.

To link to this article DOI: http://dx.doi.org/10.1007/s10546-016-0126-0

Publisher: Springer

All outputs in CentAUR are protected by Intellectual Property Rights law, including copyright law. Copyright and IPR is retained by the creators or other copyright holders. Terms and conditions for use of this material are defined in the End User Agreement.

www.reading.ac.uk/centaur 
Central Archive at the University of Reading

Reading's research outputs online 


\title{
1 Spatial and temporal variability of the concentration field from
}

\section{2 localized releases in a regular building array}

\author{
E.V.Goulart • O. Coceal • S.Branford • T.G.Thomas • S.E.Belcher
}

\begin{abstract}
Spatial and temporal fluctuations in the concentration field from an ensemble of continuous point-source releases in a regular building array are analyzed from data generated by direct numerical simulations. The release is of a passive scalar under conditions of neutral stability. Results are related to the underlying flow structure by contrasting data for an imposed wind direction of $0^{\circ}$ and $45^{\circ}$ relative to the buildings. Furthermore, the effects of distance from the source and vicinity to the plume centreline on the spatial and temporal variability are documented. The general picture that emerges is that this particular geometry splits the flow domain into segments (e.g. "streets" and "intersections") in each of which the air is, to a first approximation, well mixed. Notable exceptions to this general rule include regions close to the source, near the plume edge, and in unobstructed channels when the flow is aligned. In the oblique $\left(45^{\circ}\right)$ case the strongly three-dimensional nature of the flow enhances mixing of a scalar within the canopy leading to reduced temporal and spatial concentration fluctuations within the plume core. These fluctuations are in general larger for the parallel flow $\left(0^{\circ}\right)$ case, especially so in the long unobstructed channels. Due to the more complex flow structure in the canyon-type streets behind buildings, fluctuations are lower than in the open channels, though still substantially larger than for oblique flow. These results are relevant to the formulation of simple models for dispersion in urban areas and to the quantification of the uncertainties in their predictions.
\end{abstract}

Keywords Concentration fluctuations • Direct numerical simulation • Urban dispersion

\section{Introduction}

E.V.Goulart • S.Branford $\bullet$ S.E.Belcher

Department of Meteorology, University of Reading, Reading, UK.

e-mail: e.v.goulart@ufes.br

O.Coceal

National Centre for Atmospheric Science (NCAS), Department of Meteorology, University of Reading, Reading, UK.

T.G.Thomas

Engineering and the Environment, University of Southampton, University of Southampton, SO17 1BJ, UK.

Present address

E.V.Goulart

Department of Environment Engineering, Federal University of Espírito Santo,Vitória, 29075-910, Brazil 
Urban dispersion models generally cater for two different types of applications: (i) urban air quality, in which sources are distributed in space, (ii) emergency response, when sources are generally localized. The modelling needs posed by the latter are quite distinct from those of the former; in particular, the prediction of concentration levels and fluctuations in the neighbourhood of a release is obviously of importance. This presents a greater challenge for operational urban dispersion models to reproduce detailed concentration patterns accurately, especially close to a localised release. Much of the uncertainty is due to the spatial and temporal fluctuations in the flow and hence in the concentration field. Over flat ground, the early wind tunnel work of Fackrell and Robins (1982) showed that, for a localised release, most of the fluctuations arise from meandering of the instantaneous plume and that the relative concentration fluctuation decays with downstream distance from the source. These results were subsequently reproduced by large-eddy simulations (LES) performed by Sykes and Henn (1992) and Xie et al. (2004, 2007). The scaled field and wind tunnel experiments of Davidson et al. (1995, 1996) demonstrated the impact of a group of idealised cubical buildings of uniform height on mean and fluctuating concentrations. They showed that the mean vertical extent of the plume increased and the root-mean-square (r.m.s.) fluctuations within the plume decreased, relative to a control plume over flat terrain. The direct numerical simulations (DNS) of Branford et al. (2011), which simulated dispersion from a passive scalar point source within an array of cubes, was able to reproduce the observed values of relative concentration fluctuations and revealed a monotonic decrease with distance from the source, as in the case of flat terrain. All these studies focused on temporal fluctuations, but the spatial variability of the concentration field is equally important in an urban context.

The flow field in urban areas is known to have a complex, three-dimensional spatial structure and to be highly unsteady in time (e.g. Coceal et al. 2006, Carpentieri et al. 2012). Using DNS data, Coceal et al. (2007a) in particular demonstrated the high degree of spatial variability of the flow in the lower canopy even for regular arrays of buildings, while Coceal et al. (2007b) showed that the temporal flow characteristics comprised both organised and random aspects. It is this unsteady, heterogeneous flow field that drives the transport of pollutants through and out of the urban canopy. Hence, the processes controlling dispersion in the urban environment are intimately connected with the structure and dynamics of the flow field (Goulart 2012, Coceal et al. 2014, Belcher et al. 2015). Yet, the resulting spatial and temporal characteristics of the concentration even for a passive scalar are quite distinct 
from those of the underlying flow field. In order to quantify the level of uncertainty in predicted model concentrations it is therefore useful to document both the spatial and the temporal variability of the concentration field. Moreover, such results can be used to improve parametrizations employed in models, thereby helping to reduce the uncertainty in their predictions.

A relevant non-dimensional parameter characterizing the nature of turbulent dispersion is the ratio of the plume width, $w$, to the integral turbulence length scale, $l$ (Hunt, 1985). When this ratio $w / l$ is small turbulent eddies influence much of the plume and hence cause it to meander as a whole; when the ratio $w / l$ is large the turbulent eddies cause mixing within the plume. Much of the phenomenology of the concentration plume from localized releases can be understood from this simple point of view, supplemented by considerations of how $w$ and $l$ change spatially. First, the width of the plume $w$ depends on distance from the source; secondly, the growth rate of $w$ with distance is strongly influenced by the flow structure in the near field (which is itself determined by the building layout). "Topological" dispersion, the lateral spread caused by streamlines diverging around obstacles, causes a much more rapid initial plume growth than in the open field (Davidson et al. 1995). In an urban canopy comprised of buildings of roughly similar size distributed homogeneously (and hence neglecting isolated tall buildings and open spaces), the largest scales are of the order of the building size. Hence, a regime where $w / l>1$ is quickly attained in an urban environment. As a result there is no significant meander, except very close to the source, where the plume width is small compared to the size of the buildings. Turbulence therefore mainly causes mixing. But another major source of mixing and dispersion, on a slightly larger scale, is the flow geometry induced by the buildings. This local mean flow structure is a conspicuous additional feature compared to the open field - but how important is its effect?

This paper addresses these issues by analyzing data from previously performed DNS. After briefly outlining the DNS datasets in Sect. 2, we begin by exploring the mean flow field structure in Sect. 3. We find that this mean flow geometry is a function of wind direction, and identify generic regions of the flow with qualitatively different structures (streets, intersections, street canyons, and open channels). Distance from the source determines the relative magnitude of the turbulence scales compared to the plume width and also the relative importance of topological dispersion compared to turbulent mixing. In Sect. 4 we document 
the spatial variability in different streets and intersections in an integral sense by computing the spatial r.m.s. fluctuation, and also characterize the temporal variability in terms of the temporal r.m.s. In Sects. 5 and 6 we look at the local variation of concentration with horizontal and vertical locations, establishing different general classes of behaviour linked with the type of box (street canyon, street channel or intersection), wind direction and distance from the source. Conclusions are given in Sect. 7.

\section{Numerical datasets}

The DNS that generated the datasets analyzed here is described in Branford et al. (2011). The domain set-up is shown in Fig. 1 and involves a regular array of cubical obstacles of height $H$. The domain size is $16 H \times 16 H$ in the horizontal and $8 H$ in the vertical - see Fig. 1 for a plan view of the domain. Here we analyze data from two runs in which the wind direction is at $0^{\circ}$ and $45^{\circ}$ to the cube array.

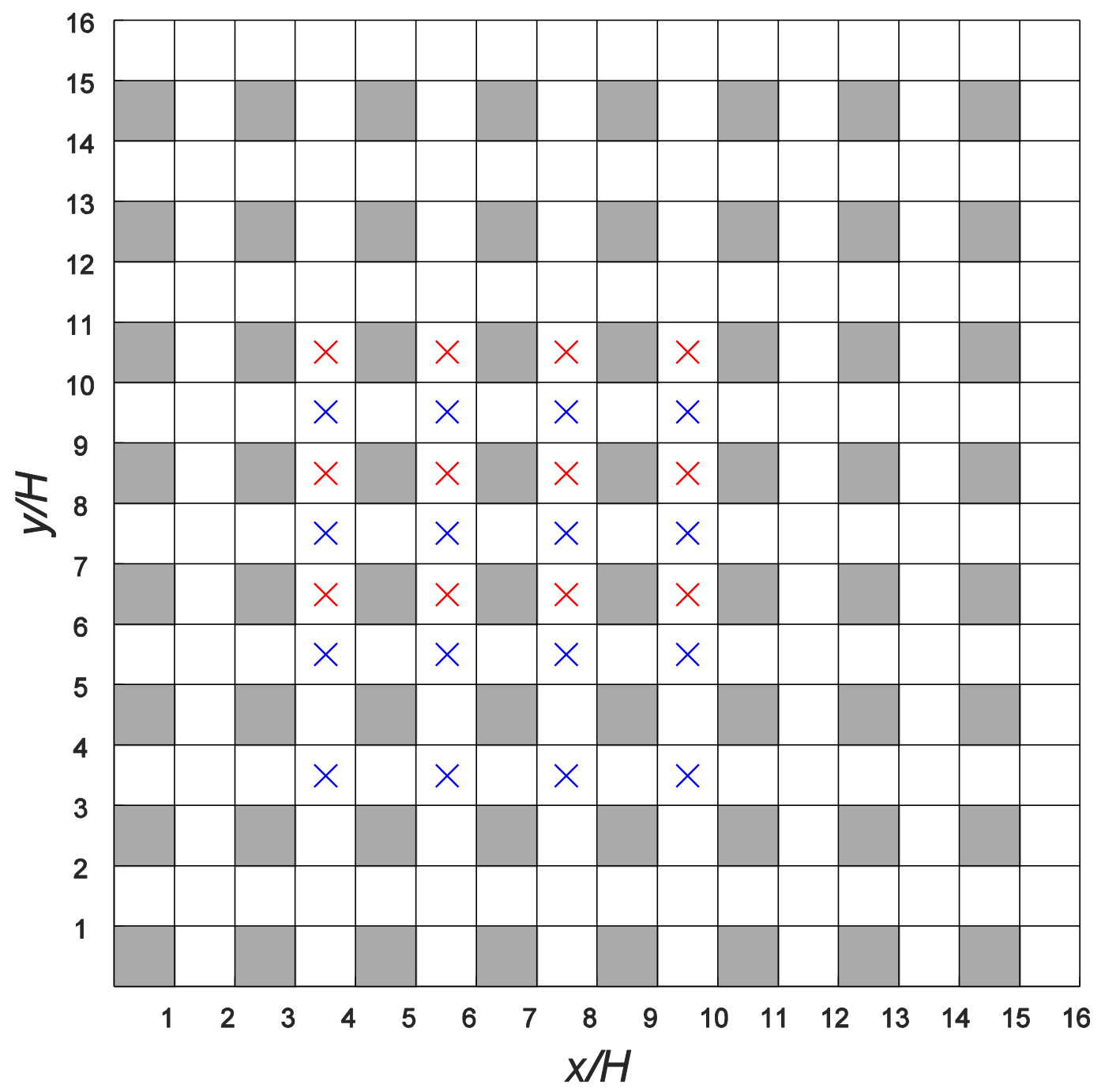


Fig 1 Plan view of the computational domains for a forcing direction of $0^{\circ}$ (left to right) and $45^{\circ}$ (along the secondary diagonal) to a regular array of cubes. Grey squares represent building positions and white squares represent streets and intersections. Red and blue crosses indicate source locations for the $0^{\circ}$ run and the $45^{\circ}$ run respectively.

The simulations were conducted under conditions of neutral stability and fully rough turbulent flow. The imposed boundary conditions were periodic in the horizontal directions, free-slip at the domain top and no-slip on the bottom and all cube surfaces. The Reynolds number based on the velocity at the top of the domain and the cube height was typically between 4750 and 7000 . While this is much less than Reynolds numbers at full scale, it is comparable to typical Reynolds numbers achieved in many wind-tunnel experiments. Numerical tests showed that a uniform grid resolution of $H / 32$ was sufficient, producing flow and concentration statistics that agreed with test runs at double the resolution $(H / 64)$ to within a few percent (Branford et al. (2011)).

A non-dimensional time scale characterizing the turnover time of eddies shed from the cubes can be defined as $T=H / u_{\tau}$, where $u_{\tau}$ is the wall friction velocity. The simulations were run with a timestep of $0.00025 T$, which is much smaller than any dynamically significant time scale. Each run was spun up for a duration of approximately $200 T$ to allow fully-developed turbulence conditions. Statistics were then collected and averages computed over an interval of approximately $75 T$ for the $0^{\circ}$ run and $100 T$ for the $45^{\circ}$ run. These averaging times are sufficient to produce statistics from which robust features may be inferred, although some residual asymmetry in flow and concentration patterns may still be apparent where none would be expected for an infinite averaging time.

Dispersion of a passive scalar released continuously and at a steady rate from an ensemble of point sources close to the ground (at $z=0.0625 H$ ) within the array was investigated; the source locations for each flow direction are indicated in Fig. 1. For each run the sources are placed in equivalent locations relative to cubes, as indicated by the crosses, so that they form an ensemble of equivalent and simultaneous releases; ensemble averaging can therefore be performed to provide a larger statistical sample, equivalent to extending the time series of an individual release but at a substantially reduced computational cost. In view of the periodic boundary conditions in the flow, a sponge layer is applied to the scalar field around the domain to prevent the scalar from re-entering the domain. The scalar is allowed to freely escape at the top of the domain. 
A major difference between dispersion over open terrain and in urban areas is the role of the mean flow field in controlling the large-scale horizontal transport of scalar in the latter. The mean flow structure is itself determined by the building geometry and the wind direction. Figure 2 shows mean streamlines projected in a horizontal plane at $z=0.5 H$ for the two flow directions of $0^{\circ}$ and $45^{\circ}$. From these streamline plots it is clear that the flow structure is strongly dependent on wind direction and also varies in different regions, e.g. streets and intersections.

In Fig. 2a the flow is aligned along one of the streets and the streamline structure is consequently nearly rectilinear and planar along the open channel formed of consecutive streets and intervening intersections. There is little difference between the mean flow structure in these streets and in the middle part of the intersection. A weak lateral mean flow exists from the edge of the intersection into the side streets (Goulart, 2012). The flow structure in the side streets is completely different, being dominated by a large recirculation in the building wake, as seen in the full-scale observations of Louka et al (2000). In the present geometry the street is short and hence the recirculation at the end of the street is a dominant structure. In longer streets their influence would be restricted to a distance along the street of the order of the street width; indications of the extent of this flow structure can be inferred from the observations of Dobre et al (2005) and the numerical simulations of Soulhac et al. (2009). The residual asymmetry in the streamline pattern between the two side streets is due to the averaging time of $75 T$; a much longer averaging time would be needed to reproduce perfect symmetry. Figure $2 \mathrm{~b}$ shows the corresponding streamline pattern for $\mathrm{a}$ wind direction of $45^{\circ}$. Here the flow structure is very different in the streets and intersections. In the streets, there is a large recirculation behind the buildings while streamlines in the other half of the street channel roughly parallel to the street axis into the downstream intersection. In the intersection there is a dividing streamline along the diagonal line joining the building corners; streamlines on either side of the dividing streamline first converge onto then diverge away from it and enter the far side of streets on either side of the intersection. 
Based on the above observations, two different regions can be identified for parallel and oblique flow corresponding to two qualitatively different flow regimes in each case. For a flow angle of $0^{\circ}$ (Fig. 2a) one can differentiate between unobstructed channel-type streets and sheltered canyon-type streets (i.e. the side streets referred to in the previous paragraph). In this case 'intersections' can be counted among channel-type streets. For $45^{\circ}$ there is instead a distinction between streets and intersections. The juxtaposition of these elemental regions establishes the large-scale flow topology in the network of streets, which in turn determines the broad features of the plume resulting from a localized release of passive scalar.

(a)

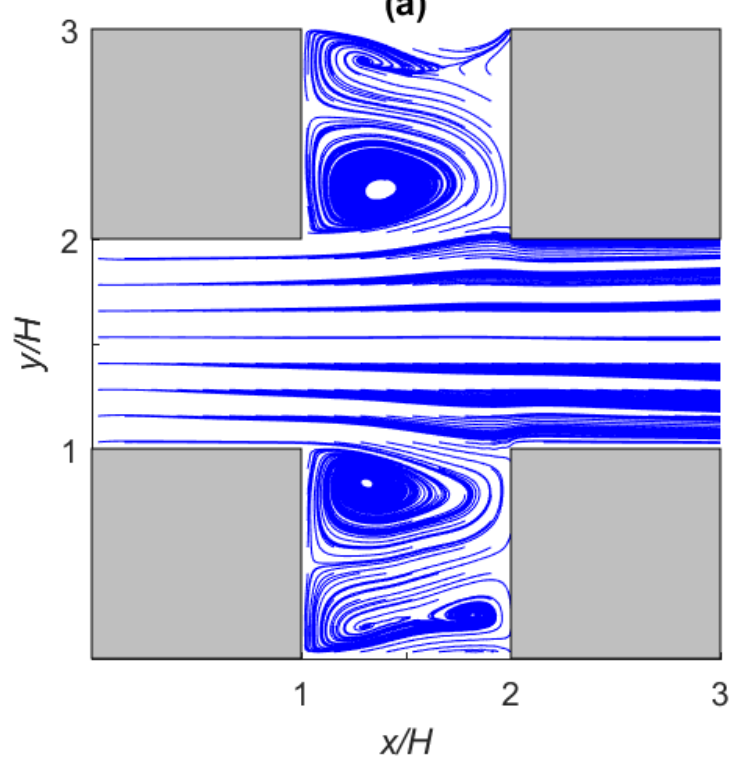

(b)

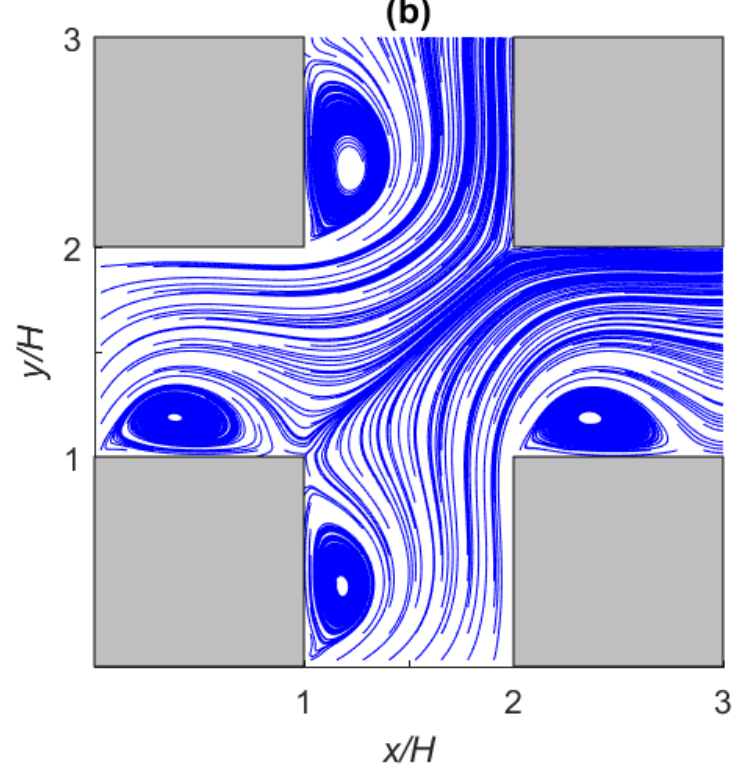

Fig 2 Plan view of horizontal streamlines of the temporally-averaged flow field at $\mathrm{z}=0.5 \mathrm{H}$ for a flow direction of (a) $0^{\circ}$ (b) $45^{\circ}$.

(a)

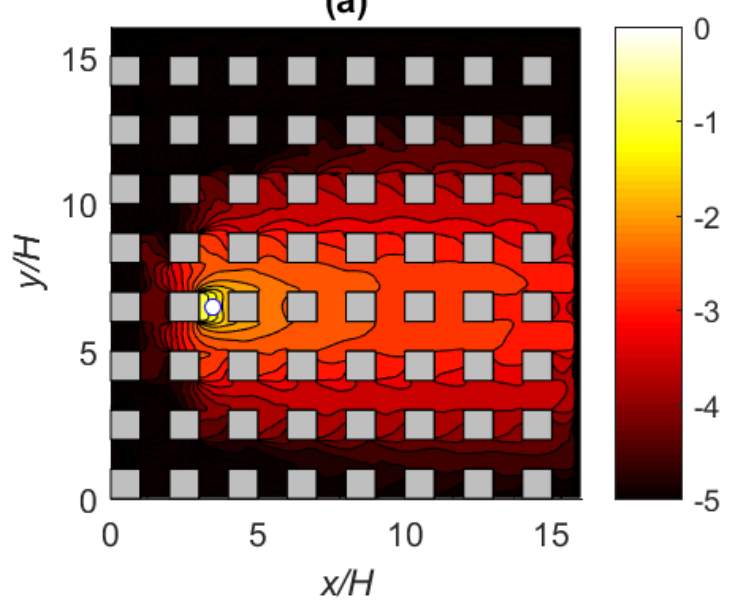

(b)

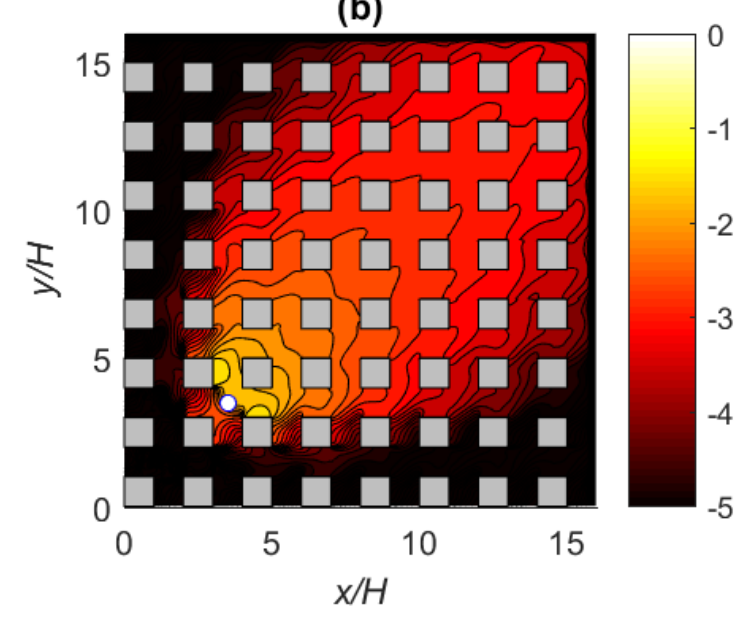


Fig 3 Mean concentration contours at $\mathrm{z}=0.5 \mathrm{H}$ for a wind direction of (a) $0^{\circ}$ (b) $45^{\circ}$. The common logarithm of normalized concentration is plotted. The ensemble-averaged source location is $(3.5,6.5)$ in (a) and $(3.5,3.5)$ in (b), as indicated by the white disks.

Figure 3 shows the resulting mean concentration pattern for the two wind directions for the source release configurations shown in Fig. 1, ensemble-averaged over the set of equivalent release locations. The ensemble averaging is achieved by shifting the origin of the coordinate system for each source such that the effective source location in each case is at $(3.5,6.5)$ for the $45^{\circ}$ simulation and at $(3.5,3.5)$ for the $45^{\circ}$ simulation, as indicated by the white disks in Fig. 3. The concentration field from all the sources are then averaged to produce the ensemble-averaged mean concentration patterns shown over an effective domain of the same size as the computational domain, as in Fig. 3. Hence, there are twelve ensemble members contributing to the average for the $0^{\circ}$ case and sixteen emsemble members for the $45^{\circ}$ case. There are fewer ensemble members contributing to the average near the edges, which implies larger statistical uncertainty in those regions. This ensemble averaging procedure was applied to produce all the concentration results presented herein (Figs. 3 to 9). The main observation is that the plume is wider for a flow angle of $45^{\circ}$ owing to enhanced lateral dispersion. This is a direct result of the very different flow topology as shown in Fig. 2b, hence the term 'topological dispersion' to describe this process (Davidson et al. 1995). Further features of the dispersion pattern are discussed in detail in Coceal et al. (2014). Despite its considerable influence on the overall, large-scale, structure of the scalar plume, the mean flow field on its own tells us little about the small-scale features of the concentration pattern. This depends on local properties of the turbulence and the size and location of the plume. These in turn depend on geometrical factors such as distance from the source, wind direction as well as the local flow geometry within the streets and intersections. The influence of these factors can be observed qualitatively from the contour plots in Fig. 3. Hereafter we focus on quantifying this small-scale variability of the plume in relation to these geometrical factors.

\section{Spatial and temporal concentration fluctuations within streets and intersections - Volume averages}

The question we address in this section is the following: how variable in space and time is the concentration in each street and intersection? We first pose the question of the spatial variability in an integral sense. Such an integral measure can be defined as the standard deviation of spatial fluctuations of the mean concentration over a suitable spatial average. For 
the present purposes a suitable domain of spatial averaging is the volume of each street and intersection. The spatial standard deviation fluctuation is then given by

$$
\sigma_{S}=\sqrt{\left\langle(\bar{c}-\langle\bar{c}\rangle)^{2}\right\rangle},
$$

where the overbar denotes a time average and the angled brackets denote a space average over the volume of each box. A temporal standard deviation can be defined as

$$
\sigma_{T}=\sqrt{\left\langle\overline{\left.c^{\prime} c^{\prime}\right\rangle}\right.}
$$

where the prime denotes an instantaneous fluctuation from the local time average. In the following plots, both $\sigma_{T}$ and $\sigma_{S}$ are normalized by the box-averaged concentration ${ }_{C *}=\langle\bar{c}\rangle$. Furthermore, results have been ensemble-averaged for the multiple sources shown in Fig. 1.

Figure 4 shows normalized values of $\sigma_{S}$ and $\sigma_{T}$ within the array for the two flow directions of $0^{\circ}$ and $45^{\circ}$ for streets and intersections for locations of sampling boxes along three different transects around the plume centreline. Note that the $\mathrm{x}$-axis in Fig. $4 \mathrm{a}$ to $4 \mathrm{~d}$ refer to the $\mathrm{x}$ ccordinate of the relevant location. Hence, for the $45^{\circ}$ case a factor of $\sqrt{2}$ should be taken into account to refer to actual distances from the source location. To complement this picture, Table 1 shows values for these quantities averaged over the whole domain. A number of general observations can be made: (i) $\sigma_{s}$ is significantly greater for $0^{\circ}$ than for $45^{\circ}$; (ii) for $0^{\circ}$ incident direction $\sigma_{S}$ is generally larger than 1, whereas for a $45^{\circ}$ direction $\sigma_{S}$ is substantially smaller than 1 in both streets and intersections; (iii) $\sigma_{T}$ is greater for $0^{\circ}$ than for $45^{\circ}$ close to the source; (iv) further from the source the value of $\sigma_{T}$ tends to approximately 0.5 for both $0^{\circ}$ and $45^{\circ}$. Taken together, these results indicate that mixing is more efficient for the oblique flow. Interestingly, greater spatial variability in the flow field is associated with smaller variability in the concentration field. This is particularly evident when comparing the value of $\sigma_{S}$ in the channel-type streets with those in the canyon-type streets and in the intersections. The reason is that a heterogeneous, three-dimensional flow field contributes to mixing.

2

(1) 
(a)

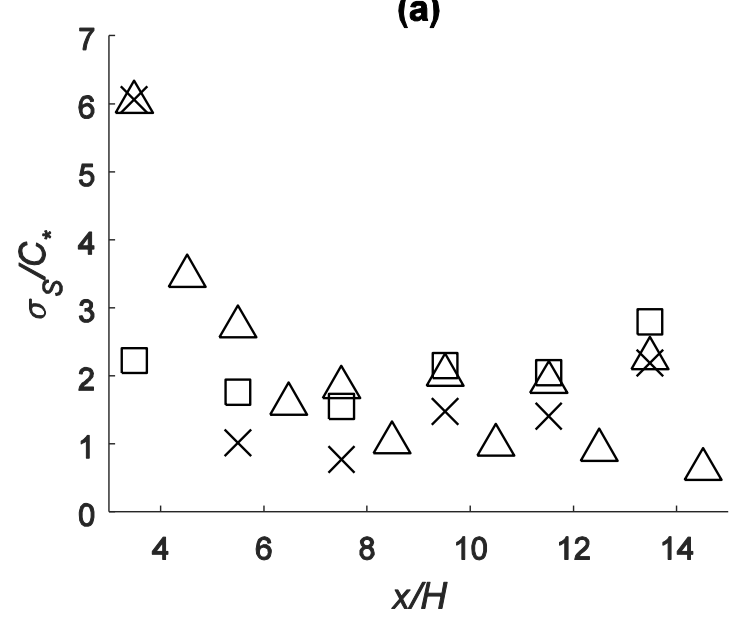

(c)

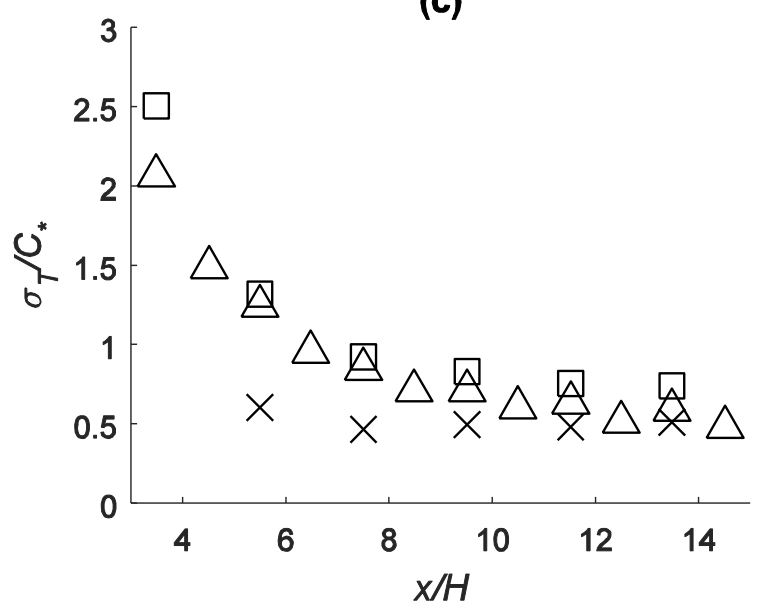

(e)

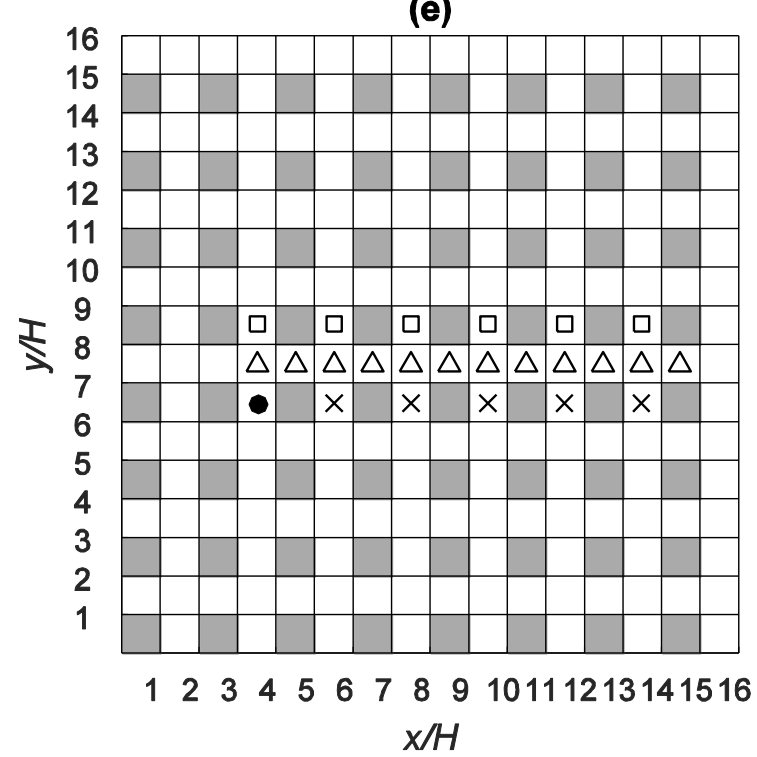

(b)

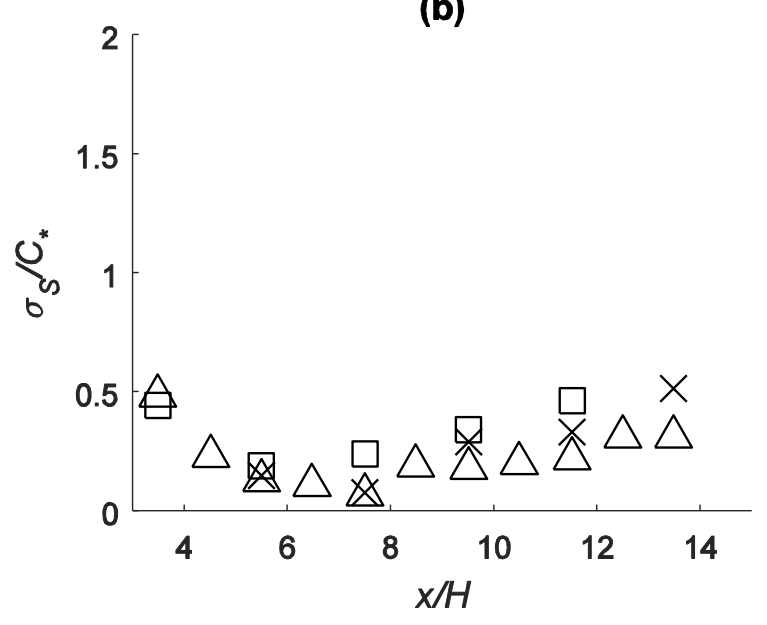

(d)

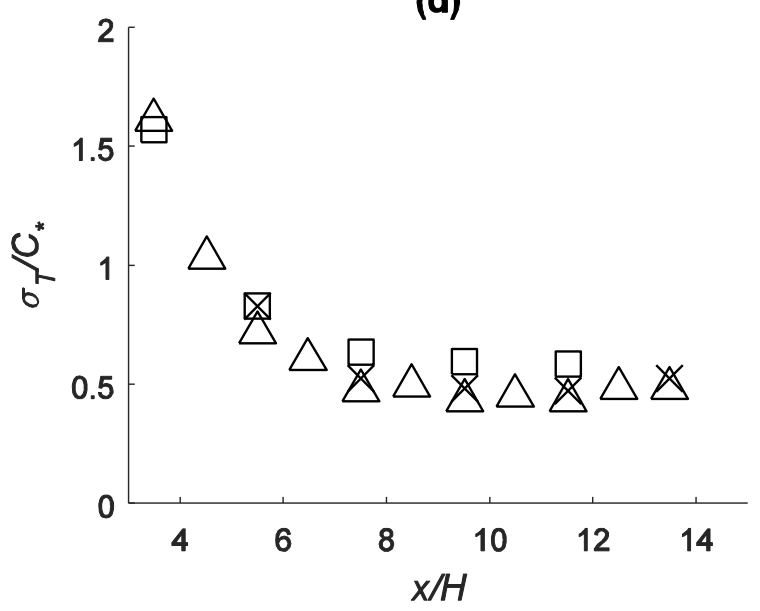

(f)

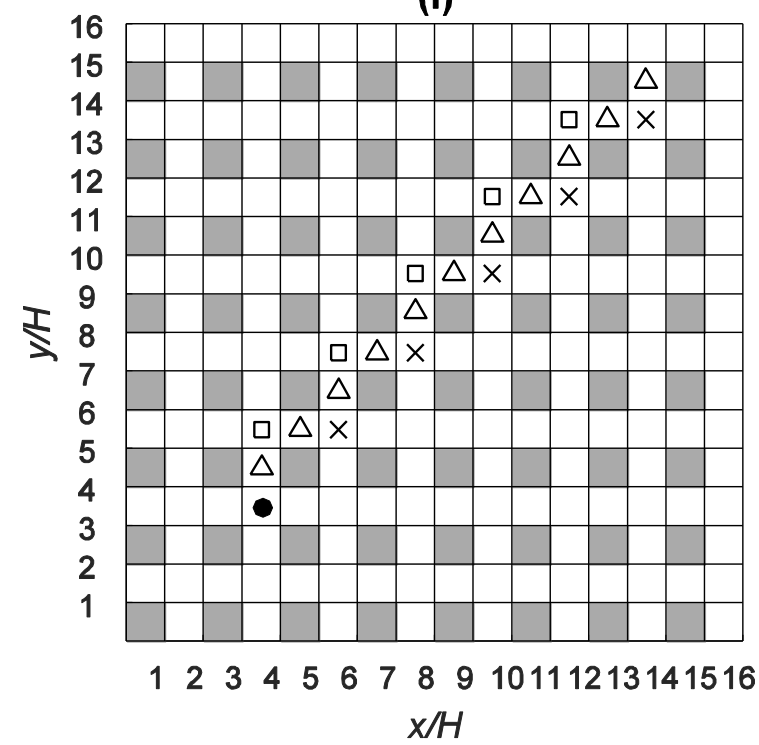

257 Fig 4 (a) Spatial $\left(\sigma_{S}\right)$ and (c) temporal $\left(\sigma_{T}\right)$ concentration fluctuations within the array for $0^{\circ}$. (b) Spatial and

258 (d) temporal concentration fluctuations within the array for $45^{\circ}$. (e) \& (f) Crosses, triangles and squares indicate 259 locations of sampling volumes along three different transects for $0^{\circ}$ and $45^{\circ} . \sigma_{S}$ and $\sigma_{T}$ are normalized by the 
264

ensemble-averaged mean concentration, $C_{*}$. The black dots indicate the ensemble-averaged source locations. Note the different vertical scales in the plots.

\begin{tabular}{lrr}
\hline Location & $\sigma_{S} / C_{*}$ & $\sigma_{T} / C_{*}$ \\
\hline Street, 45 & 0.3 & 0.9 \\
Intersection, 45 & 0.4 & 1.0 \\
${\text { Channel-type street, } 0^{\circ}}^{\circ}$ & 2.1 & 2.0 \\
Canyon-type street, $0^{\circ}$ & 1.3 & 1.7
\end{tabular}

Table 1: Normalized spatial $\left(\sigma_{S} / C_{*}\right)$ and temporal $\left(\sigma_{T} / C_{*}\right)$ concentration fluctuations for streets and intersections, averaged over the whole domain.

\section{Local variability of concentration within streets and intersections - Horizontal structure}
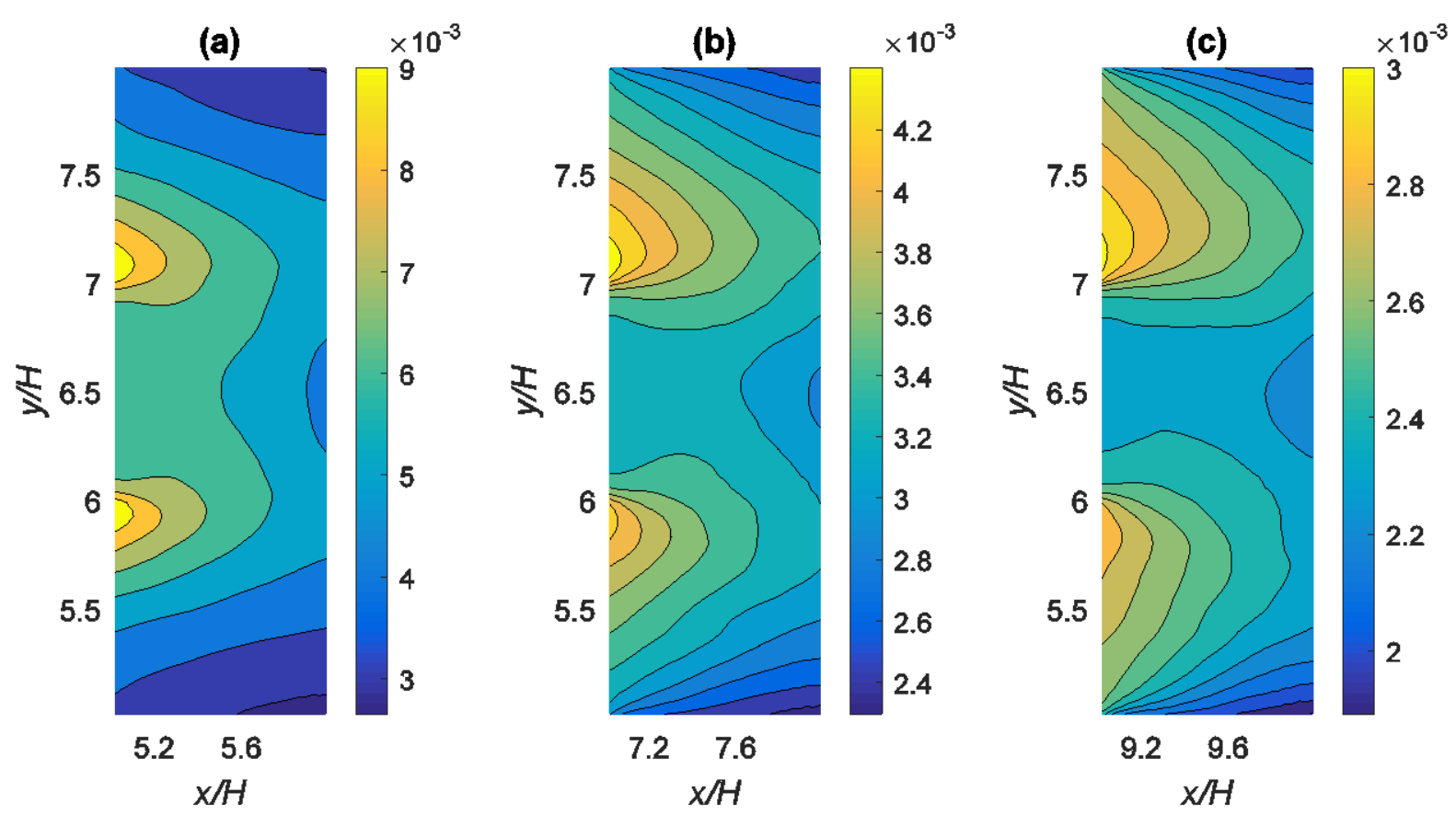


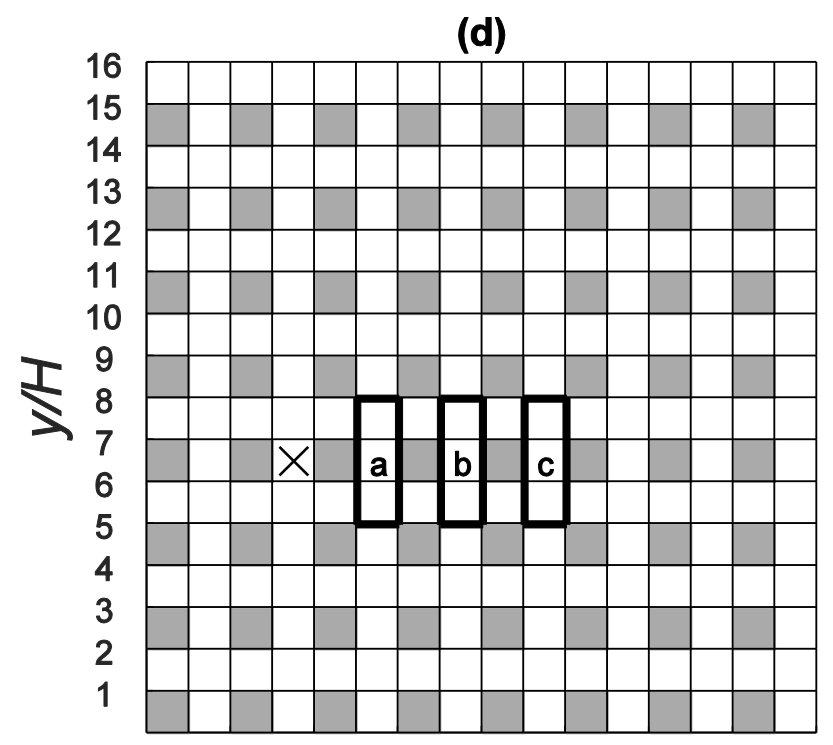

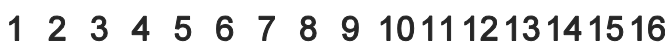

Fig 5 (a), (b) and (c) Plan view of the time-averaged concentration for a flow angle of $0^{\circ}$ in sections denoted by $\mathrm{a}, \mathrm{b}$ and c respectively, (d) Locations of sampled sections (denoted by $\mathrm{a}, \mathrm{b}, \mathrm{c}$ ) and ensemble-averaged source location (denoted by a cross). Concentrations are normalized by the concentration at the ensemble-averaged source location.

We now focus on the local variability of the concentration within streets and intersections. Figures 5-7 show a plan view of the time-averaged concentration field in selected streets and intersections at $z=0.5 \mathrm{H}$. The patterns at different heights are generally very similar, except close to the building tops. Since the concentration patterns for the whole domain at that height are shown in Fig. 3 we now choose to focus on specific regions that afford a comparison between qualitatively different dispersion patterns. For a flow direction of $0^{\circ}$, scalars are mostly advected along the open channels on either side of the source (which is located in a canyon-type street - see Fig. 1). Figure 5 shows the mean concentration patterns in three different regions spanning three adjacent streets one, two and three streets downstream from the release location. Qualitatively similar patterns exist for corresponding regions further downstream (not shown). As for the streamline pattern in Fig. 2a, the slight asymmetry in these concentration patterns between the two side streets is due to the averaging time of $75 \mathrm{~T}$. The concentration levels in the two channel-type streets are much higher than that in the canyon-type street between them. Within the latter, the concentration is slightly higher on the upstream side. The spatial concentration variation (difference between maximum and minimum concentration) in the canyon-type street is $41 \%$ of the maximum value in that street one street downstream from the release location. This decreases 
to $21 \%$ and $14 \%$ respectively two and three streets downstream from the source. Larger variations are found in the channel-type streets, with corresponding spatial fluctuations of $66 \%, 37 \%$ and $29 \%$ at the same location of one, two and three streets downstream from the source. This is again a consequence of the greater mixing in the canyon-type streets due to the more complex flow structure.

Figure 6 shows concentration patterns in selected intersections for a flow angle of $45^{\circ}$, with figure 6a showing the concentration pattern for the second intersection along the centreline from the ground source. A variation of $25 \%$ of the maximum concentration is found within this particular intersection. The bottom left corner of the intersection has the largest concentration due to the accumulation of material in the nearby wake regions behind the building just upstream. Since the selected intersection lies on the plume centreline the concentration is symmetric about the diagonal across the intersection.

Figure $6 \mathrm{~b}$ shows the concentration field in the fourth intersection downstream from the source location, also along the centreline. Now further from the source, the variation of the concentration across the intersection reduces as the plume becomes more well-mixed (Finn et al. 2010). The variation in concentration within the intersection is reduced to $15 \%$ and there is a nearly monotonic decrease in the concentration variation with respectively $50 \%, 25 \%$, $13 \%$ and $15 \%$ in the first, second, third and fourth intersection from the source. This decrease in the spatial variability with distance from the source mirrors the monotonic decrease in temporal fluctuations observed by Branford et al. (2011) and Coceal et al. (2014). We note once again the slight asymmetry in the concentration patterns in Fig. 6a and 6b, due to the averaging time of $100 T$. 
(a)
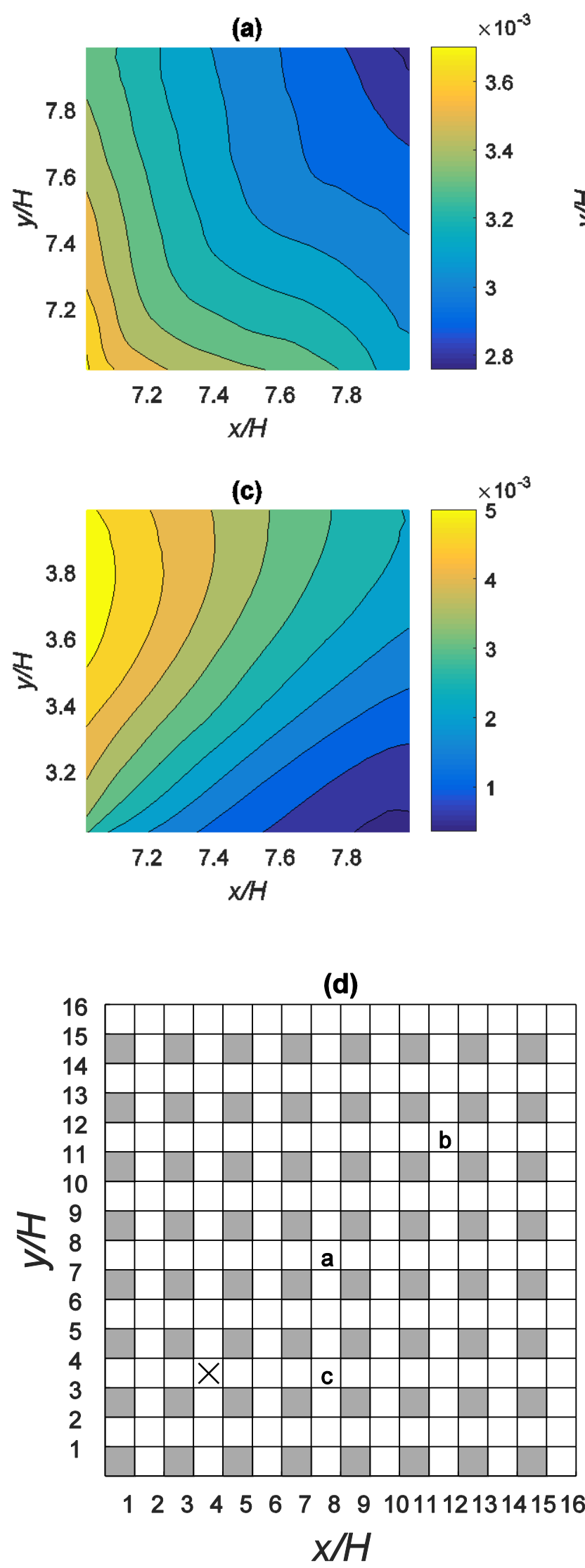

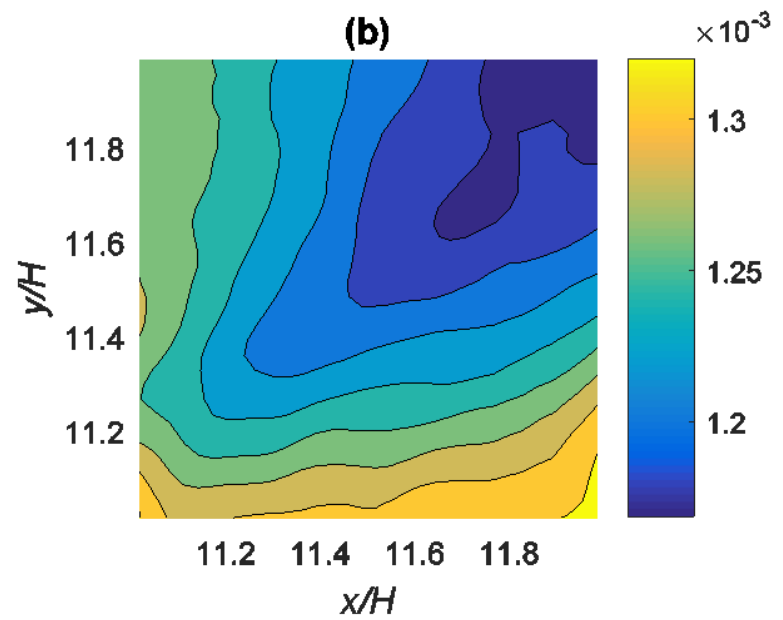


Fig 6 (a), (b) and (c) Plan view of the time-averaged concentration at $\mathrm{z}=0.5 \mathrm{H}$ for a flow angle of $45^{\circ}$ at intersections denoted by a, b and c respectively, (d) Locations of sampled intersections. Concentrations are normalized by the concentration at the ensemble-averaged source location, denoted by a cross.

Figure $6 \mathrm{c}$ shows the concentration field in an intersection at the edge of the plume. Here the flow is partly from a street with clean air and partly from a street with polluted air, leading to a greater degree of spatial variability than for intersections within the plume. So, while it is a fair approximation to consider the air in intersections within the plume to be well-mixed, this approximation breaks down at the edges of the plume.

For comparison, the concentration variation within four streets at $z=0.5 \mathrm{H}$ is shown in Fig. 7a,d, with street locations shown in Fig. 7e. Figures $7 \mathrm{a}$ and $7 \mathrm{~b}$ show concentration patterns in streets on either side of the second intersection from the source along the plume centreline. The concentration variation in these streets is between $20-30 \%$ of the maximum value. Even further from the source, the concentration variation remains at this level for the rest of the array. The concentration in these streets reaches local peaks at the upstream end of the streets and close to the recirculation areas.

Figure $7 \mathrm{c}$ shows the concentration within a street immediately downstream of the release location; in this near-field street, the variation of mean concentration is $71 \%$ of the highest concentration (hence, the ratio of minimum to maximum concentration in that sreet is 0.29 ). The highest concentration is found close to the wake of the building, due to substantial amounts of material being trapped in the recirculation region; there is however more material at the street entrance (on the left of the box) near to the ground, due to direct advection from the source (not shown). The mean concentration pattern is different in the next street downstream (Fig. 7d). The highest concentration does not now appear in the recirculation area but in the upper side of the street, just after the preceding intersection; the variation in concentration is $43 \%$ of the maximum. 
(a)

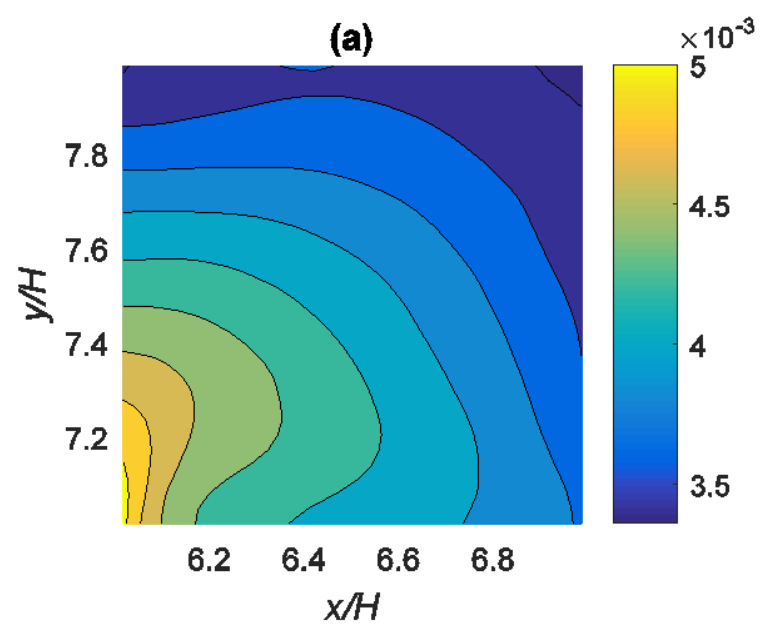

(c)

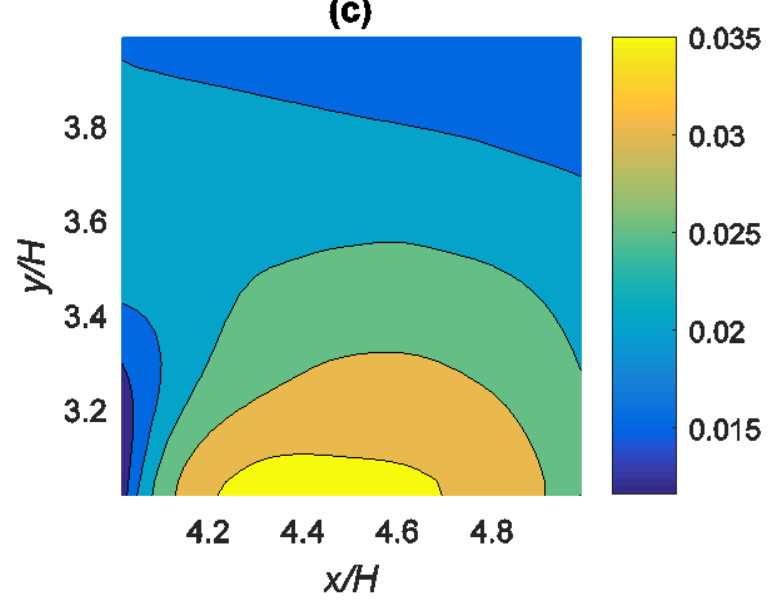

360

(e)

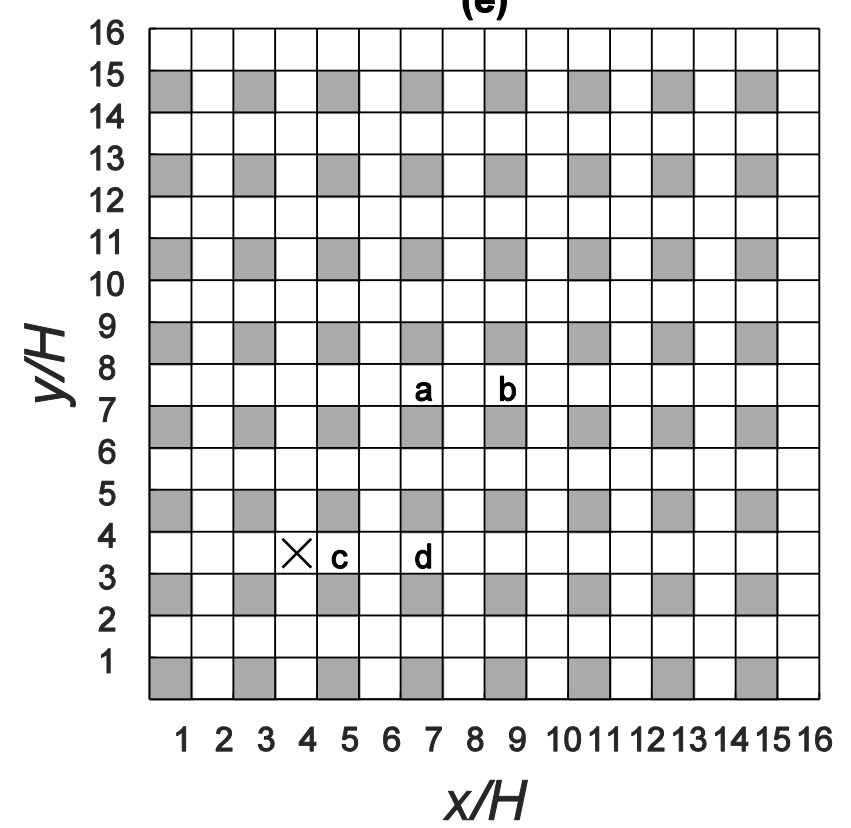

Fig 7 (a), (b), (c) and (d) Plan view of the time-averaged concentration at $\mathrm{z}=0.5 \mathrm{H}$ for a flow angle of $45^{\circ}$ at streets denoted by a, b, c and d respectively, (e) locations of sampled streets. Concentrations are normalized by the concentration at the ensemble-averaged source location, denoted by a cross. 
Next we consider the vertical variability of the mean concentration at different locations in a box. Figures 8 and 9 show vertical concentration profiles in boxes located at increasing distances from the source along the centreline and immediately adjacent to it for flow directions of $0^{\circ}$ and $45^{\circ}$ respectively. Profiles were sampled at the centre, upwind edge and downwind edge through the middle of each box for $0^{\circ}$, and at the centre, upwind corner and downwind corner of each box for $45^{\circ}$. These locations were chosen to give a wide range of variation in the concentration profiles. The volume-averaged concentration in each box is also plotted for comparison.

For a flow direction of $0^{\circ}$, the behaviour is distinctly different within the two types of streets (canyon-type and channel-type), especially close to the source. In the first, the concentration is reasonably constant with height and profiles sampled at different horizontal locations are closer to each other. In the first canyon after the source in Fig. 8a there is a sharp decrease near the top of the array. For the street in the channel there is a substantially greater degree of variability both with height and with horizontal location (Fig. 8b). The concentration profiles for the second and third canyon after the source in Fig. 8c and 8e are closer together and the decrease of the concentration near the top of the array is less pronounced. The concentration is more well-mixed with height. Along the channel streets (Fig. 8d and 8f) the concentration varies less horizontally and vertically as the distance from the source increases. 
(a)

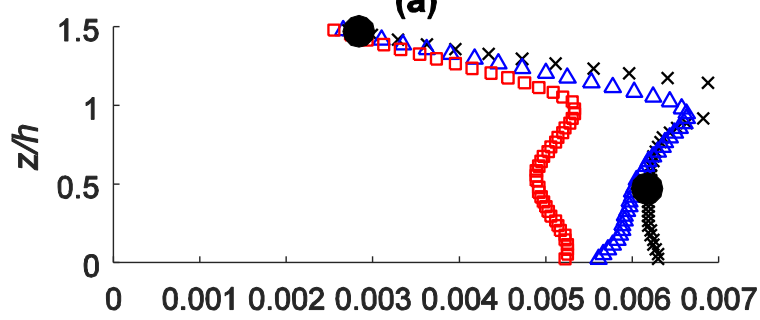

C.

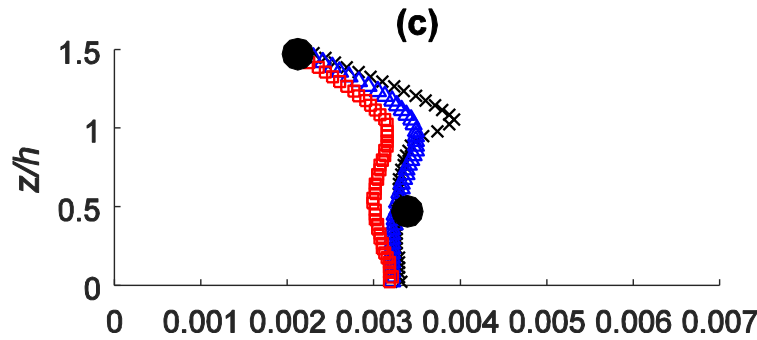

C.

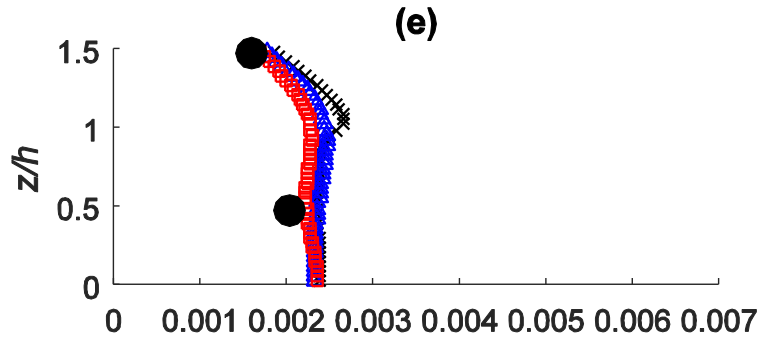

C.

(g)

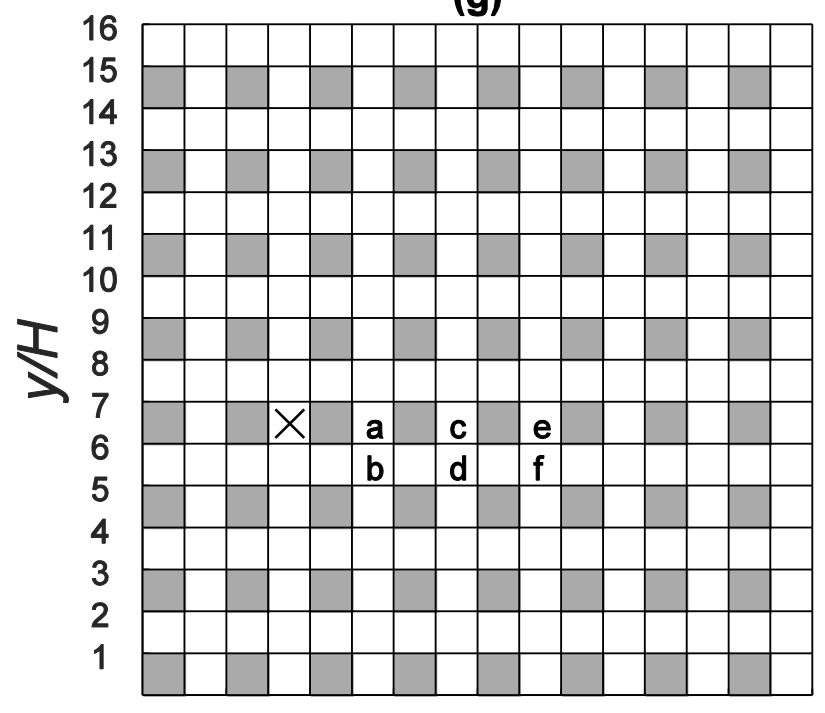

1233456788910111213141516

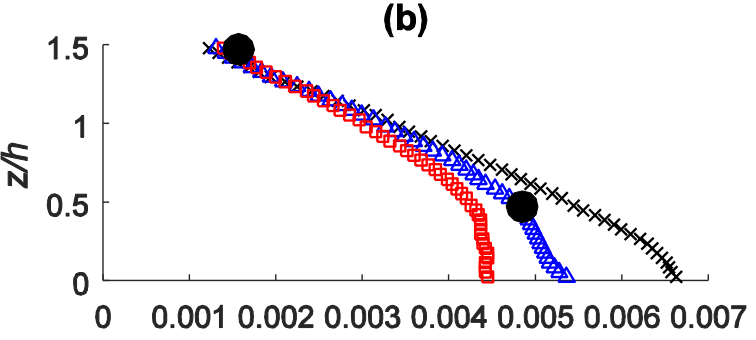

C.

(d)

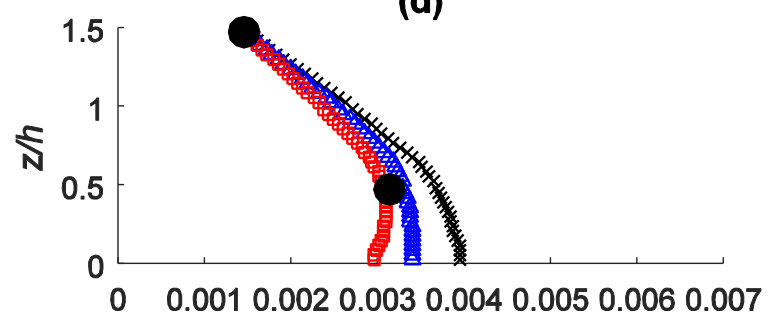

C*

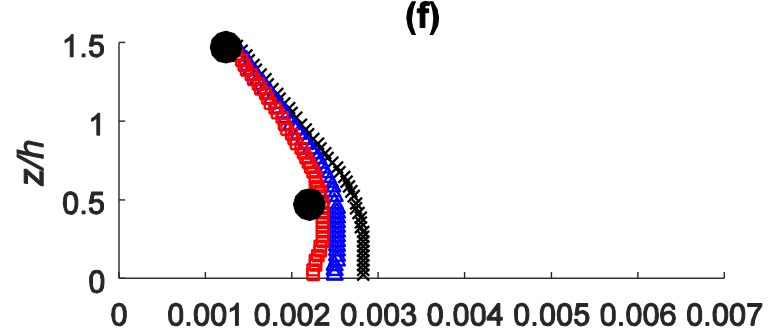

C*

Fig 8 (a), (b), (c), (d), (e) and (f) Vertical profiles of ensemble-averaged mean concentration for a flow direction of $0^{\circ}$ at sampling locations denoted by a, b, c, d, e and f respectively. Symbols (x), ( $\Delta$ ) and (口) respectively represent the concentration sampled at the upwind edge, centre and downwind edge within each box and (•) represents the box-averaged ensemble-averaged mean concentration $C_{*}$. (g) Sampling locations. 
Concentrations are normalized by the box-averaged concentration at the ensemble-averaged source location, denoted by a cross.

398

399

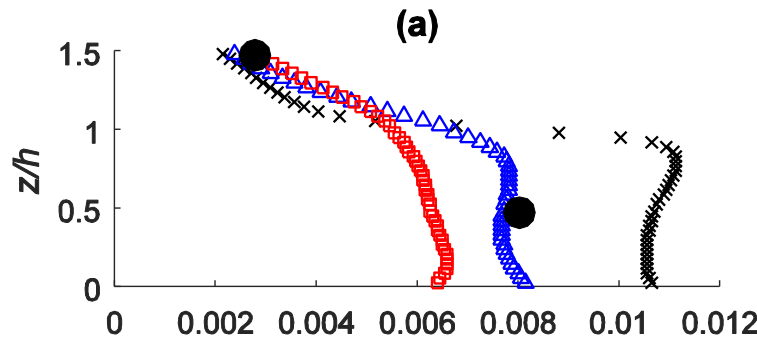

C.

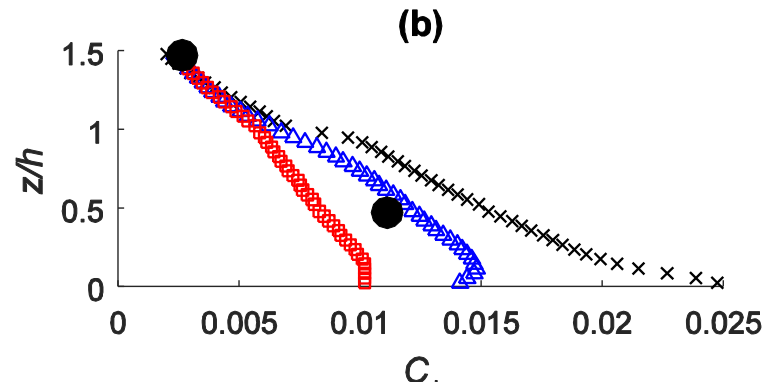

(c)

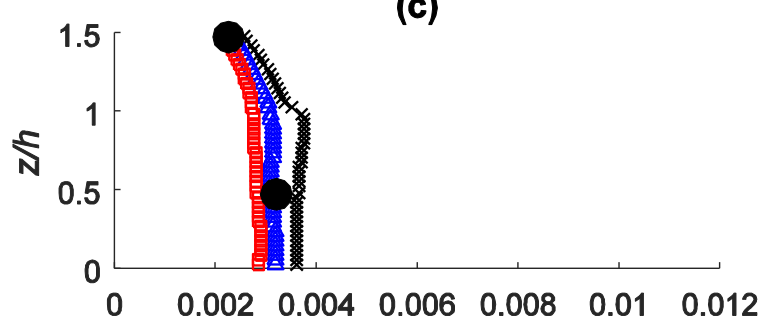

C*

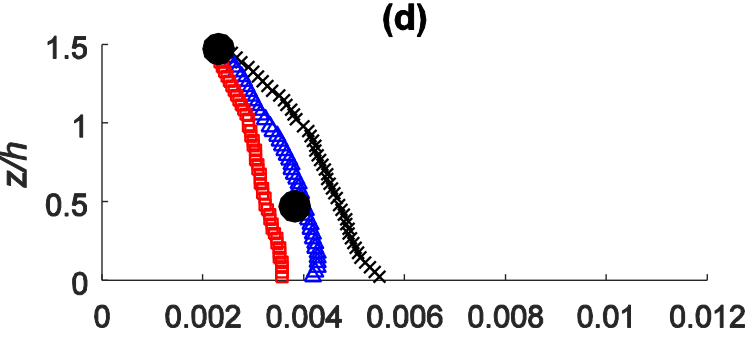

(e)

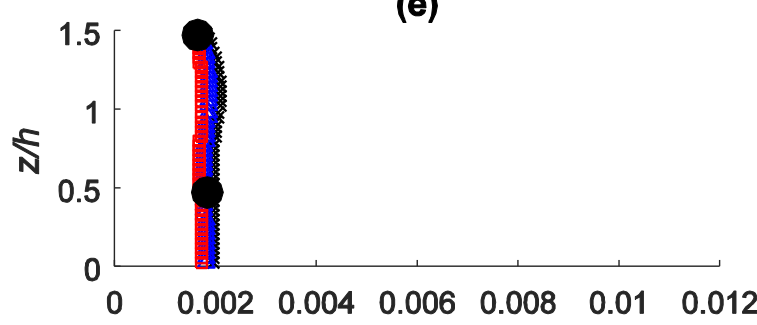

400

C.

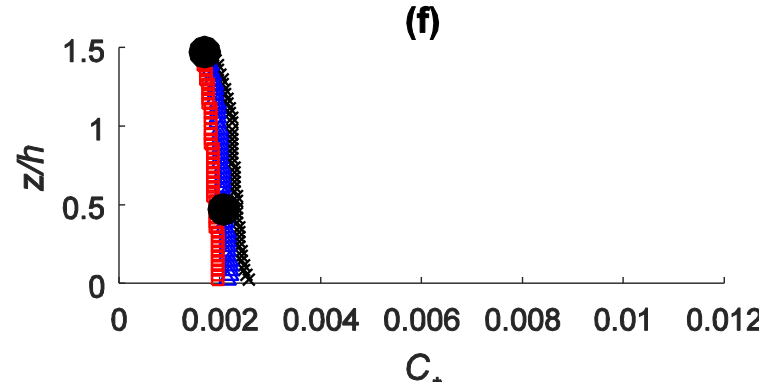


(g)

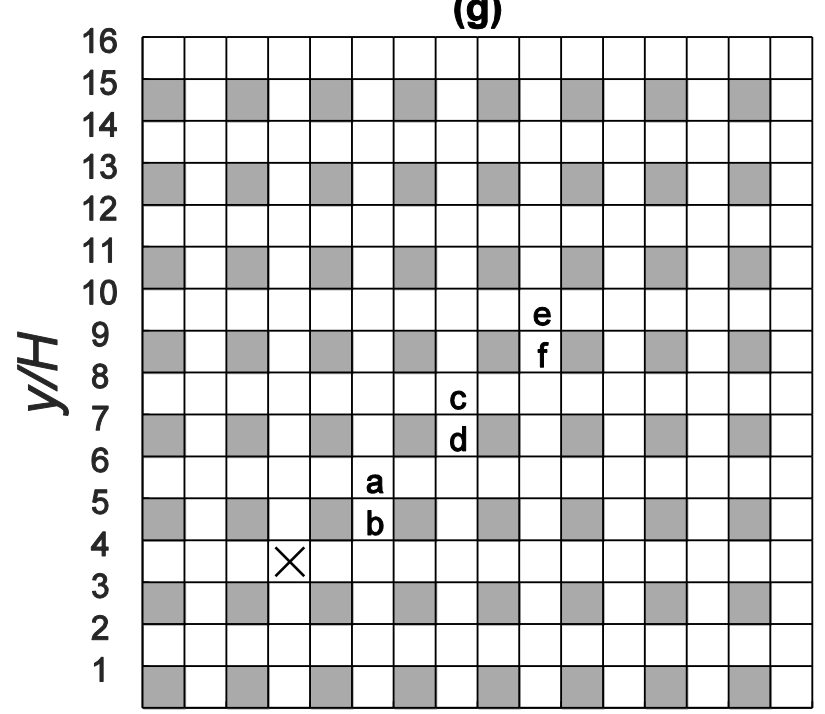

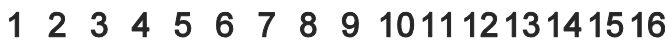

401

402

403

404

405

406

407

408

409

410

411

412

413

414

415

416

417

$x / H$

Fig 9 (a), (b), (c), (d), (e) and (f) Vertical profiles of ensemble-averaged mean cncentration for a flow direction of $45^{\circ}$ at sampling locations denoted by a, b, c, d, e and f respectively. Symbols (x), ( $\Delta$ ) and ( $\square$ ) respectively represent the concentration sampled at the upwind corner, centre and downwind corner within each box and $(\bullet)$ represents the box-averaged ensemble-averaged mean concentration $C_{*}$. (e) Sampling locations. Concentrations are normalized by the concentration at the ensemble-averaged source location, denoted by a cross.

Figure 9 shows corresponding vertical profiles of the mean concentration in three intersections along the plume centreline at increasing distances from the source for a flow direction of $45^{\circ}$. The concentration in the first intersection downstream from the source location is nearly constant with height over most of the depth of the canopy, but with a rapid decrease near the top of the array (Figure 9a). The variation with horizontal location is larger, of the order of $20 \%$ of the mean. Figure 9c shows corresponding profiles for the second intersection downwind of the source location. The profiles are now much closer together and more constant with height, with a much reduced concentration gradient near the canopy top. By the third intersection downwind (Fig. 9f) the profiles have collapsed and show a constant value with height, with little discernible difference between the concentration within the canopy and that immediately above. This indicates that there is no net scalar transfer from the canopy to above, and the plume is well mixed not only within the canopy, but also just above the buildings. There is a similar general tendency towards greater spatial homogeneity in streets too. The street adjacent to the first intersection, shown in Fig. 9b, is somewhat atypical in that there is a substantially greater degree of vertical variability. 


\section{Conclusions}

425 We have shed light on the spatial and temporal variability of the concentration field from a point source release and its dependence on factors such as distance from the source, flow direction and local flow structure. The external flow direction has a strong effect on the flow structure and dynamics and hence on the mechanisms of dispersion within the canopy. For an oblique flow a three-dimensional structure is created, enhancing mixing of the scalar within the canopy. In contrast, for a parallel flow scalar is mostly channelled along the unobstructed streets. Behind the buildings (street canyons) the air is mixed more effectively than along the channels and the spatial variability of concentration is therefore reduced. In general, the temporal and spatial concentration fluctuations are larger for the parallel flow, especially along the channel-type streets. Due to the complex flow structure behind the building, the temporal and spatial concentration fluctuations are lowest in the street canyons. When the external flow is oblique to the array, the three-dimensionality of the resulting flow structure, as well as enhanced turbulence levels, lead to reduced temporal and spatial concentration fluctuations. In that situation the concentration is well mixed within the canopy along the core of the plume. At the edge of the plume the temporal and spatial concentration fluctuations increase and the vertical concentration profile is not constant with height. These results can be summarized in the following simplified picture: to a first approximation a scalar is almost always well-mixed in the vertical, and generally so in the horizontal, except near the source and the edges of the plume and in the unobstructed channels that occur when the flow is aligned to a long street. These findings are useful for simplified predictive models that employ a well-mixed assumption (e.g. Hamlyn et al. 2007; Belcher et al. 2015). Moreover, the computations of spatial and temporal variances given here represent a useful estimate of minimum uncertainty levels to be attributed to models that only predict mean or spatially averaged concentrations, or to localized experimental measurements.

Finally, it is important to point out the potential pitfall of over-generalization based on the present results. By necessity, the scope of this work is limited on several accounts and many questions remain, particularly in connection with the effect of varying the set-up and parameters prescribed. For example, it is pertinent to ask how the results differ for different source locations. This is difficult to answer in any general way without actually performing a potentially large number of simulations. But it is reasonable to expect that large differences might be confined to the vicinity of the source location, since a localized release is likely to 
457 be mixed rather rapidly over a larger effective initial area in the turbulent urban environment.

458 Another key consideration is the effect of wind direction. The two examples studied reveal important differences between dispersion patterns when the flow is aligned with the streets and when it is at an oblique angle. Which of these cases is more generic? Given that it is rare in practice for the wind to be perfectly aligned to a street and that, in any case, the wind direction typically varies by tens of degrees in the atmosphere, the oblique flow case is almost certainly more characteristic of real urban flows. But the issue still remains of how sensitive the details of the dispersion pattern are to the wind direction. Finally, the present building geometry is comprised of cubical buildings of the same size arranged in a regular pattern at a particular spacing. Would similar results apply in a real city, given the heterogeneity of the building and street geometry? We hope that the present study will inspire similar analyses for more realistic urban configurations, perhaps using large-eddy simulations.

Acknowledgements Elisa V. Goulart's PhD was funded by the National Council for Scientific and Technological Development (CNPq), Brazil. Simon Branford was supported by the University of Reading Research Endowment Trust Fund. Omduth Coceal gratefully acknowledges funding from the Natural Environment Research Council (NERC) through their National Centre for Atmospheric Science (NCAS) under grant no. R8/H12/83/002 and from the Engineering and Physical Sciences Research Council (EPSRC contract number EP/K040707/1).

Conflict of Interest: The authors declare that they have no conflict of interest.

\section{References}

Belcher SE, Coceal O, Goulart EV, Rudd AC, Robins AG (2015) Processes controlling dispersion in urban areas. J Fluid Mech 763:51-81

Branford S, Coceal O, Thomas T, Belcher SE (2011) Dispersion of a point source release of a passive scalar through an urban-like array for different wind directions. Boundary-Layer Meteorol 139: 367-394

Carpentieri M, Hayden P, Robins A (2012) Wind tunnel measurements of pollutant turbulent fluxes in urban intersections. Atmos Environ, 46:669-674

Coceal O, Dobre A, Thomas TG, Belcher SE (2007a) Structure of turbulent flow over regular arrays of cubical roughness. J Fluid Mech 589:375-409

Coceal O, Thomas TG, Belcher SE (2007b) Spatial variability of flow statistics within regular building arrays. Boundary-Layer Meteorol 125:537-552 
Coceal O, Thomas TG, Castro IP, Belcher SE (2006) Mean flow and turbulence statistics over groups of urbanlike cubical obstacles. Boundary-Layer Meteorol 121:491-519

Coceal O, Goulart EV, Branford S, Thomas TG, Belcher SE (2014) Flow structure and near-field dispersion in arrays of building-like obstacles. J Wind Eng Ind Aerodyn 125:52-68

Davidson MJ, Mylne KR, Jones CD, Phillips JC, Perkins RJ (1995) Plume dispersion through large groups of obstacles a field investigation. Atmos Environ 29:3245-3256

Davidson MJ, SnyderWH, Lawson RE,Hunt JCR (1996) Wind tunnel simulations of plume dispersion through groups of obstacles. Atmos Environ 30:3715-3725

Dobre, A, Arnold, SJ, Smalley, RJ, Boddy, JWD, Barlow, JF, Tomlin, AS, \& Belcher, SE (2005) Flow field measurements in the proximity of an urban intersection in London, UK. Atmos Environ 39:4647-4657

Fackrell JE, Robins AG (1982) Concentration fluctuations and fluxes in plumes from point sources in a turbulent boundary layer. J Fluid Mech 117:1-26

Finn D, Clawson K, Carter R, Rich J, Biltoft C, Leach M. (2010) Analysis of urban atmosphere plume concentration fluctuations. Boundary-Layer Meteorol 136:431-456

Goulart EV (2012) Flow and dispersion in urban areas. PhD Thesis, University of Reading, UK

Hamlyn D, Hilderman T, Britter R (2007) A simple network approach to modelling dispersion among large groups of obstacles. Atmos Environ 41(28):5848-5862

Hunt, JCR (1985) Turbulent diffusion from sources in complex flows. Annu Rev Fluid Mech, 17:447-485

Louka, P, Belcher, SE \& Harrison, RG (2000) Coupling between air flow in streets and the well-developed boundary layer aloft. Atmos Environ, 34:2613-2621

Soulhac L, Garbero V, Salizzoni P, Mejean P, Perkins R (2009) Flow and dispersion in street intersections. Atmos Environ 43(18):2981-2996

Sykes RI, Henn DS (1992) Large-eddy simulation of concentration fluctuations in a dispersing plume. Atmos Environ 17:3127-3144

Xie Z-T, Hayden P, Voke PR, Robins AG (2004) Large-eddy simulation of dispersion: comparison between elevated source and ground-level source. J Turbul 5:1-23 

flow over a rough wall. Atmos Environ 41:3395-3406 